\title{
Utilisation d'un altimètre (ALTUS) destiné à la quantification des dépôt/érosion en domaine littoral pour l'étude des corrélations avec les caractéristiques de vagues et les interfaces de dépôt
}

\author{
Ability of an acoustic echosounder (Altus) intended to quantify bed level \\ variations in coastal areas to correlation studies with wave features \\ and surficial sediment deposits
}

\author{
PHILIPPE BASSOULLET, ROMARIC VERNEY, YOUEN KERVELLA, STÉPHANE KERVELLA*, \\ HERVÉ JESTIN, GUILLAUME VOINESON \\ Ifremer - Centre de Brest, DYNECO/Physed, B.P. 70, 29280 Plouzané, France \\ * Ifremer LERPC, Place Gaby Coll, B.P. 5, 17137 L’Houmeau, France
}

Tél : +33 (0)2 982243 44, Fax : +33 (0)2 982248 64, e-mail : pbassoul@ifremer.fr

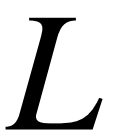

'évaluation quantitative fine des séquences d'érosion/dépôt des sédiments superficiels est primordiale pour comprendre les processus et établir des bilans sédimentaires en domaine littoral. Le système ALTUS a été conçu, depuis plus d'une décennie, pour répondre à cet objectif. Les possibilités de mesures offertes par cet altimètre (mesures à court terme ou sur plusieurs mois) sont particulièrement appropriées pour permettre une validation des modèles numériques hydro-sédimentaires.

L'intégration récente d'une carte mémoire de grande capacité a permis d'optimiser ce système. En effet, il est bien établi que la houle est un paramètre clé pour l'érosion des sédiments en zones intertidales, gouvernant ainsi le transport sédimentaire. Le système ALTUS, outre l'enregistrement du suivi altimétrique, permet d'accéder aux caractéristiques de vagues (hauteur, période significative, frottement sur le fond...)

Des corrélations peuvent ainsi être établies entre une érosion constatée et la dynamique du milieu (vagues, impacts anthropiques...)

Dans ce papier il est également fait état de tests en laboratoire réalisés dans le but d'évaluer la capacité d'ALTUS à détecter une modification relativement «brutale » des caractéristiques physiques du sédiment des dépôts superficiels.

$Q$

uantitative evaluation of erosion/deposition events of surficial sediments is crucial to understand the processes and establish sedimentary budgets in coastal areas. The ALTUS system was designed for more than a decade, to meet this objective. The possibilities offered by this altimeter (short-term or several months measurements) are particularly fitted for validation of sedimentary transport numerical models.

The recent integration of a large capacity memory card has allowed to optimize the system. Indeed, it is well established that wave activity is one of the key parameters for the erosion and hence govern sediment transport in intertidal/shallow water areas. The ALTUS system, in addition to bottom level variation recordings, allows access to wave characteristics (wave height, significant period, bottom shear stress...)

Correlations can be established between a recorded erosion and dynamics (waves, human impact...)

In this paper it is particularly reported laboratory tests carried out in order to assess the ability of ALTUS to detect relatively « rough » changes of the physical characteristics of surficial sediment deposits.

\section{I $\square$ INTRODUCTION}

L'étude de la dynamique sédimentaire en zones littorales nécessite l'acquisition in situ et en continu d'un certain nombre de paramètres relatifs d'une part à l'hydrodynamisme du milieu et d'autre part aux propriétés intrinsèques du sédiment contrôlant sa remise en suspension, son transport et son dépôt.
La mise en évidence de ces mouvements sédimentaires passe par une évaluation quantitative fine de ces séquences d'érosion/dépôt des sédiments superficiels afin d'approcher des bilans sédimentaires locaux ou régionaux. La compréhension de la dynamique de ces dépôts nécessite classiquement des relevés à fréquence variable (bimensuelle, mensuelle...) qui ne permettent cependant pas d'approcher les phénomènes haute fréquence (échelle tidale par exemple). Seule une appro- 
che par enregistrements, en continu et à haute fréquence, de l'évolution du niveau de sédiment permet de déterminer les processus mis en jeu pour l'érosion ou l'accrétion des sédiments. Ces divers processus peuvent être : la variation du courant de marée, les houles courtes et longues, les effets de dessèchement du sédiment superficiel en relation avec l'exposition plus ou moins longue au soleil sur les estrans exondés à basse mer et, bien entendu, les modifications des sédiments superficiels dues aux activités biologiques (bioturbation par exemple), ou encore l'effet de drainage.

Depuis plus de dix années la collaboration menée entre Ifremer et la société NKE -Micrel a permis l'élaboration du système ALTUS ("ALTimètre à Ultrasons Submersible »), un sondeur enregistreur acoustique autonome pour le suivi de l'altimétrie du sédiment de surface et, par là même, pour l'évaluation des séquences de dépôt/érosion, avec un échantillonnage à haute fréquence. Les spécifications du système ALTUS sont présentées dans [1]. A court terme par exemple, ce capteur permet une quantification précise (d'ordre millimétrique) des dépôts liés à une étale de marée ; à plus long terme, sur plusieurs mois, il enregistre le suivi de l'évolution sédimentaire d'une vasière ou d'un estran sableux. De nombreuses études utilisant ALTUS ont été menées en zones littorales et estuariennes, en intertidal ou subtidal afin de valider des modèles numériques de transport de sédiments [2], [3] et [4].

De nombreux tests en laboratoire ont été récemment réalisés à Ifremer-Brest afin d'évaluer la capacité d'ALTUS (i) à détecter des faciès sédimentaires différents et (ii) à déterminer des épaisseurs de dépôts sédimentaires de caractéristiques physiques différenciées.

Dans sa version initiale, la capacité mémoire de l'appareil ne permettait pas d'enregistrer la totalité du signal. Récemment, suite à l'intégration d'une carte mémoire de plus grande capacité, il a été testé la possibilité de le faire pour avoir l'intégralité de chaque écho avec l'idée de pouvoir accéder à certaines caractéristiques (i.e. type de faciès, « état de consolidation ",...) des sédiments superficiels.

Le système permet également d'accéder aux caractéristiques de vagues (hauteur, période significative...).

Après une présentation rapide du système ALTUS et des exemples de déploiements sur le terrain et d'interprétation des résultats, ce papier traite des tests laboratoire permettant d'envisager des développements complémentaires aux données altimétriques actuelles fournies par le système.

\section{PRESENTATION DU SYSTEME ALTUS}

Basé sur le principe d'un échosondeur classique mais avec une fréquence d'émission d'ondes acoustiques de $2 \mathrm{MHz}$, le système ALTUS est constitué de deux parties : (1) le transducteur acoustique, positionné à une trentaine de centimètres au-dessus du sédiment et orienté vers le bas, (2) le module enregistreur, déporté du transducteur et relié à lui par un câble. Le temps de trajet depuis l'émission de l'onde acoustique et sa réception par le transducteur est converti en distance. Cette célérité du son dépend de la température et de la salinité du milieu. Le module comporte un capteur de pression (permettant de connaître la hauteur d'eau sur le site), l'énergie et l'électronique (Fig. 1). Un « crayon » magnétique permet de communiquer avec un PC pour la programmation et le déchargement des données.

Deux gammes de mesures transducteur-surface du sédiment sont disponibles selon les environnements à investiguer : (1) 20 à $70 \mathrm{~cm}$ (avec une précision de $\pm 2 \mathrm{~mm}$ ) et (2) 20 à $200 \mathrm{~cm}$ (avec une précision de $\pm 5 \mathrm{~mm}$ ) ; la résolution est de $0.6 \mathrm{~mm}$ dans les deux cas.

Outre l'enregistrement de la hauteur d'eau ( $<25 \mathrm{~m})$, l'ALTUS acquiert de 1 à 4 seuils de détections d'énergie acoustique reçue correspondant à la hauteur transducteur-sédiment, et ceci à la cadence désirée. Ces seuils permettent de préciser l'interface eau-sédiment et sont des indicateurs de l'état de sédimentation/consolidation des dépôts. Ainsi un dépôt frais de vase liquide sur un fond de vases plus consolidées doit pouvoir être détecté et quantifié. Afin d'optimiser ces seuils, il est nécessaire de les programmer en laboratoire, après étalonnage à partir des types de faciès sédimentaires investigués.

La fréquence d'échantillonnage maximale est de $2 \mathrm{~Hz}$, en continu ou par salves. Selon les objectifs attendus les cadences de tirs sont programmées en fonction de la capacité mémoire du module ALTUS portée maintenant à 4 Mo.
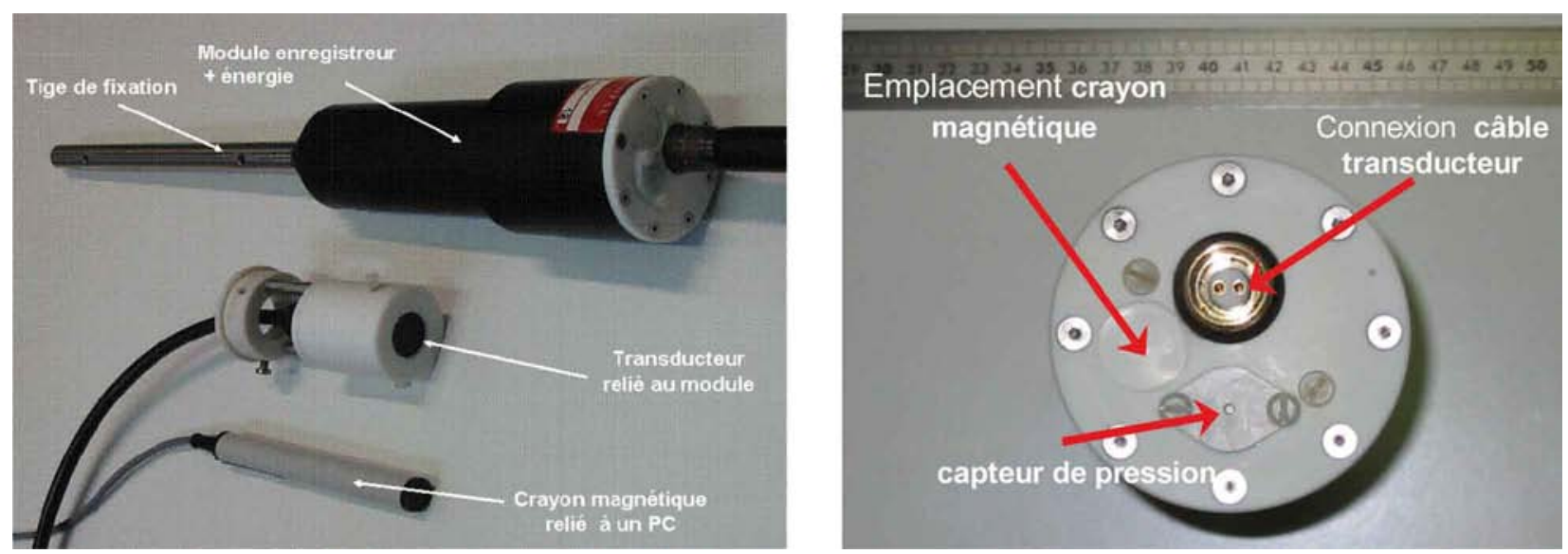

Figure 1 : Système ALTUS 
Une sonde Température - Salinité peut être placée à proximité de l'appareil pour une éventuelle correction de la célérité du son.

\section{III — EXEMPLES D'ENREGISTREMENTS A PARTIR DE DEPLOIEMENTS TERRAIN}

\section{III.1 SUIVI A MOYEN TERME DES EVOLUTIONS DE NIVEAUX DE SEDIMENT}

Des enregistrements sur plusieurs mois ont été réalisés en domaine intertidal ou subtidal, dans divers environnements tels que les bassins de Marennes-Oléron et d'Arcachon, l'estuaire de la Seine ou la baie du Mont Saint-Michel (Fig. 2)

La mise en évidence des périodes " actives » en termes de dépôt/érosion a été établie avec ce système, par exemple, pour les sédiments superficiels de la baie du Mont SaintMichel (sur l'estran médian, en secteur occidental), de même que le type de forçage correspondant. L'évolution simultanée du vent est observée à Dinard. Ainsi, dans ce cas précis (Fig. 3), l'altimètre a relevé en 2 jours des érosions de $5,5 \mathrm{~cm}$ lors d'épisodes de vents de secteur Sud. La recharge continue de l'estran est ensuite perturbée par un coup de vent de secteur Nord, décapant $3 \mathrm{~cm}$ de sédiment. Une quantification est ainsi possible selon la nature des forçages en jeu.

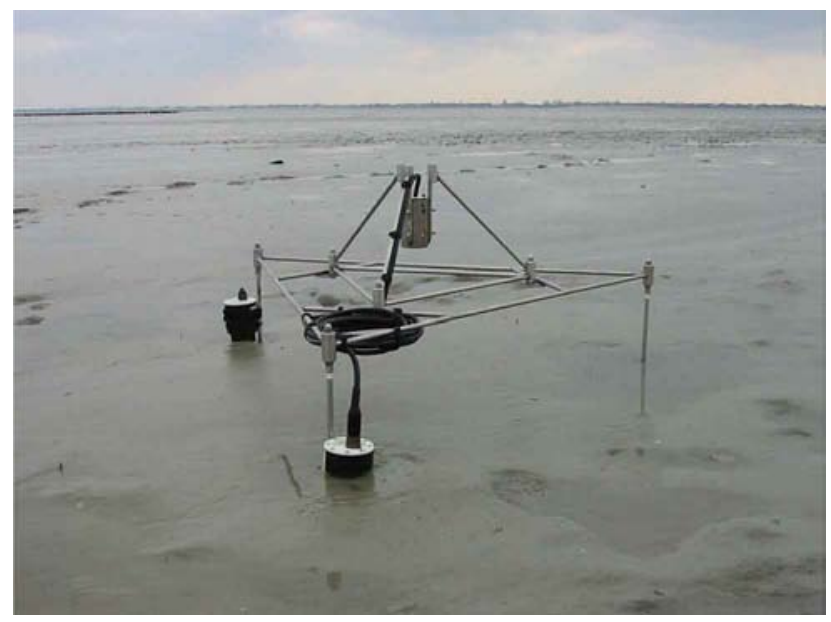

Transducteur acoustique orienté vers le bas et relié au module principal

Module principal avec capteur de pression, énergie, électronique de contrôle et centrale d'acquisition des données. Un autre module est ici dédié uniquement à l'enregistrement des hauteurs de vagues

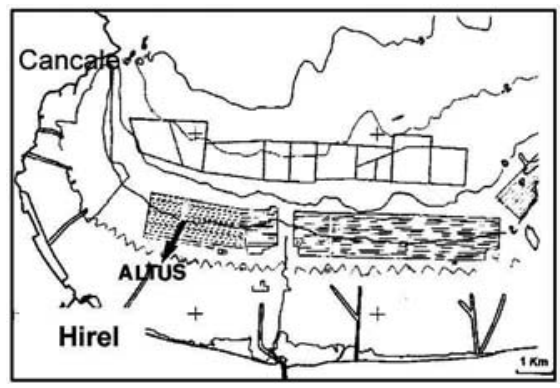

Figure 2 : Exemple de déploiement du système ALTUS en baie du Mont Saint-Michel

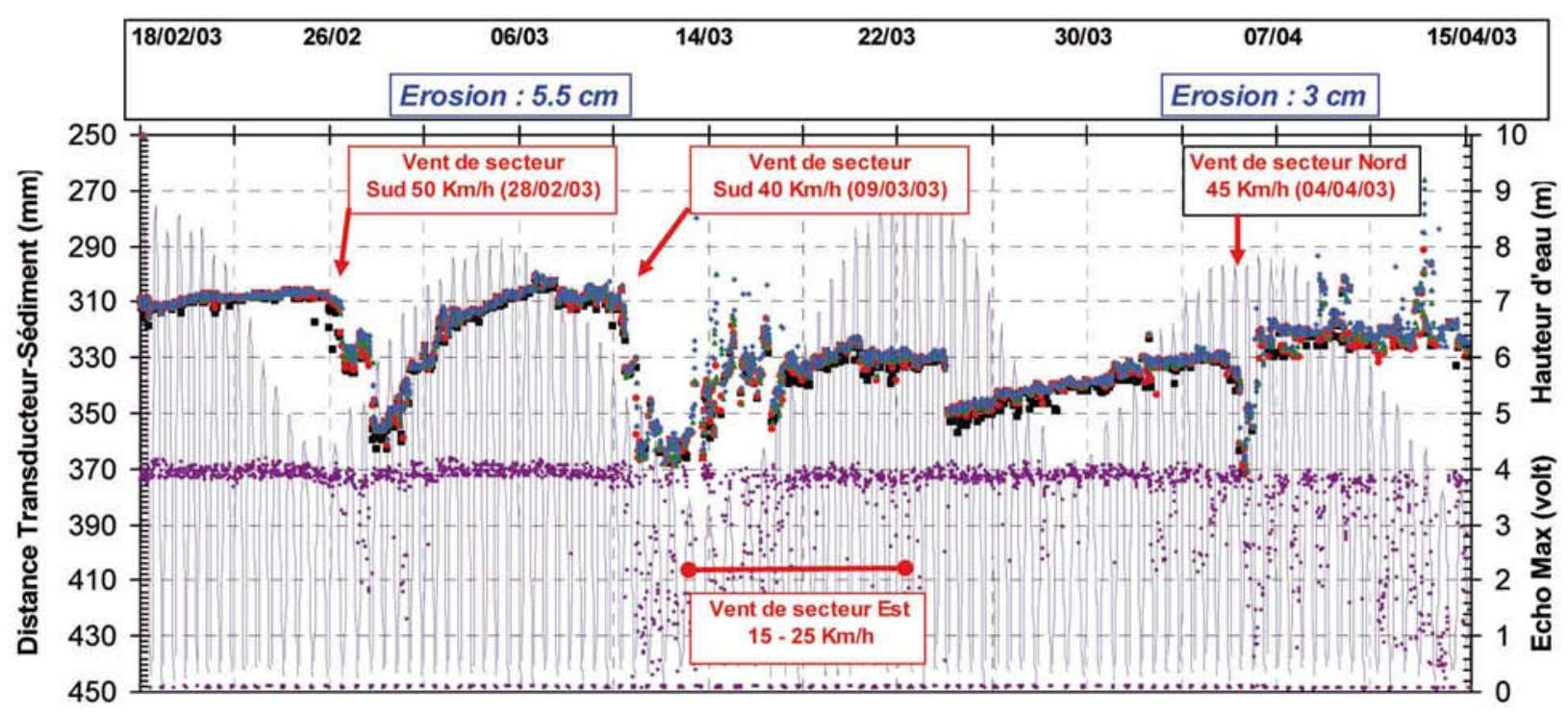

Figure 3 : Enregistrement ALTUS en baie du Mont Saint-Michel (localisation sur la carte de la figure 2) 


\section{III.2 UTILISATION DU SYSTEME ALTUS EN MODE VAGUES}

III.2.1 Traitement des données de pression : mesure des paramètres de vagues avec le Système ALTUS

Le module ALTUS est muni d'un capteur de pression à membrane pouvant fonctionner à $2 \mathrm{~Hz}$ et permettant d'estimer les hauteurs de vagues simultanément avec les enregistrements altimétriques. Afin de réduire au maximum la mémoire utilisée, la pression et l'altimétrie peuvent être enregistrées à des cadences indépendantes.

Le mode "vagues " consiste à acquérir la mesure de pression à $2 \mathrm{~Hz}$ par salves, i.e. pour un usage classique : une salve de $256 \mathrm{~s}$ toutes les 20 ou $30 \mathrm{~min}$. Le signal est ensuite traité via une routine open source développée sous Matlab ${ }^{\circledR}$ pour obtenir les principaux paramètres caractéristiques des vagues : hauteur significative $\left(H_{s}\right)$, période moyenne $\left(T_{m}\right)$ et période représentative $\left(T_{r}\right)$, vitesse orbitale représentative $\left(\mathrm{U}_{\mathrm{br}}\right)$ et tension sur le fond $\left(\tau_{\mathrm{w}}\right)$. La procédure est brièvement présentée ci-après.

Le signal de pression brut est préalablement corrigé de l'atténuation due à la profondeur. Le signal corrigé est ensuite traité par analyse spectrale pour calculer les principaux paramètres :

$$
\begin{gathered}
H_{s i g}=4 \sqrt{m_{0}}=4 \sqrt{\int S_{p}(f) d f} \cong 4 \sqrt{\sum S_{p}(f i) \Delta f i} \\
T_{m}=\frac{m_{0}}{m_{1}}=\frac{\sum S_{p}(f i) \Delta f i}{\sum f i S_{p}(f i) \Delta f i}
\end{gathered}
$$

où $\mathrm{m}_{0}$ et $\mathrm{m}_{1}$ sont respectivement les moments d'ordre 0 et 1 et $\mathrm{Sp}$ le spectre du signal de pression obtenu par transformation de Fourrier. La relation entre vitesse orbitale et pression pour chaque bande spectrale i est donnée ci-dessous :

$$
S_{u, i}=\frac{4 \pi^{2}}{T_{i}^{2} \sinh ^{2}\left(k_{i} h\right)} S_{p, i}
$$

La relation (3) permet de calculer la période et la vitesse orbitale représentatives

$$
\begin{gathered}
T_{r}=\frac{\sum S_{u i}(f i) \Delta f i}{\sum f i S_{u i}(f i) \Delta f i} \\
U_{b r}=\sqrt{2 \sum\left[\frac{4 \pi^{2}}{T_{i}^{2} \sinh ^{2}\left(k_{i} h\right)} S_{p, i} \Delta f i\right]}
\end{gathered}
$$

Supposant connue la rugosité du fond $\mathrm{z}_{0}$ (dépendant entre autres de la nature et de la forme du fond, de la présence de végétation ou d'espèces benthiques, voir [5]), il est alors possible de calculer le terme de friction $\mathrm{f}_{\mathrm{rw}}$ et la tension de cisaillement induite par les vagues, $\tau_{\mathrm{w}}$.

$$
\begin{gathered}
f_{r w}=1.39\left(\frac{U_{b r} T_{r}}{2 \pi z_{\theta}}\right)^{-0.52} \\
\tau_{w}=\frac{1}{2} \rho f_{r w} U_{b r}{ }^{2}
\end{gathered}
$$

Il est important de rappeler l'influence du choix de la valeur de la rugosité, qui peut faire varier de plusieurs ordres de grandeur la tension de cisaillement pour un même forçage vague.

\section{III.2.2 Exemple d'enregistrement de corrélations variations de hauteurs du sédiment superficiel - hauteur des vagues}

L'enregistrement des variations de hauteur du sédiment sur plusieurs mois en station 8 du secteur ostréicole de Marennes-Oléron met en évidence une brusque variation de $9 \mathrm{~cm}$ le 9 décembre 2007 (Fig. 4). A cette station le sédiment, de type vase, se dépose sur un faciès sableux. Durant plus de 2 mois, en conditions hydrodynamiques calmes, il est constaté un dépôt continu de $3 \mathrm{~cm}$ de vase. Deux épisodes de tempêtes suivent avec des hauteurs significatives de vagues notables : un épisode ponctuel dans le temps (V1), suivi quelques jours plus tard d'un épisode plus intense et de durée plus longue (V2). L'analyse des corrélations entre ces vagues et l'évolution de la hauteur du sédiment, mesurées avec l'ALTUS, permet de conclure à une certaine liquéfaction du sédiment au cours de la $1^{\text {ère }}$ tempête favorisant ainsi son érosion $(9 \mathrm{~cm})$ lors de la tempête suivante, plus marquée.

\section{TESTS EXPERIMENTAUX EN LABORATOIRE}

Ce type d'enregistrement nous a conduits à nous intéresser au matériel sédimentaire lui-même afin de rechercher les éventuelles potentialités du système ALTUS à détecter des sédiments de caractéristiques physiques différentes, à partir de l'enregistrement de la totalité du signal acoustique de retour. Pour ce faire, des carottes « artificielles » ont été reconstituées par dépôts successifs (de 5 à $20 \mathrm{~mm}$ ) de sédiments de granulométrie variée sur divers types de faciès sédimentaires (Fig. 5) ; ces alternances de dépôts ayant été observées sur le terrain.

Ainsi, les tests en laboratoire ont été menés afin de vérifier les capacités de réponse de l'ALTUS vis-à-vis (1) de la précision de la mesure du niveau de surface du sédiment, (2) de la capacité à détecter un faciès particulier (sable grossier, sable fin, vase fine et mélange de type vase sableuse) et enfin, (3) à détecter une interface franche entre deux faciès contrastés : sable fin sur sable grossier, vase sur sable et vase fine fluide sur vase compactée. L’objectif étant de rechercher si, à partir de l'enregistrement de l'intégralité du 

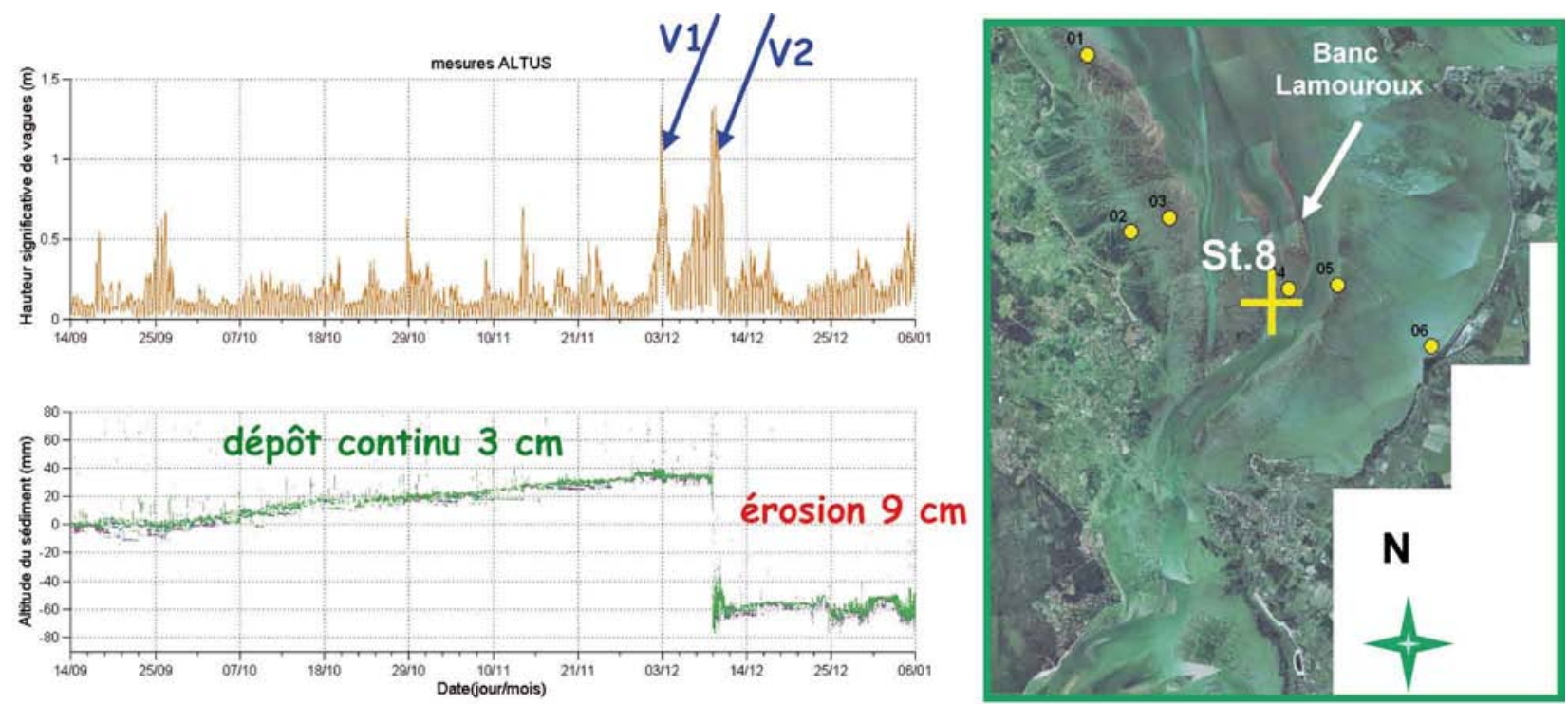

Figure 4 : Enregistrement ALTUS (environ 4 mois) en baie de Marennes-Oléron - St. 8 -(Côte Atlantique, France)
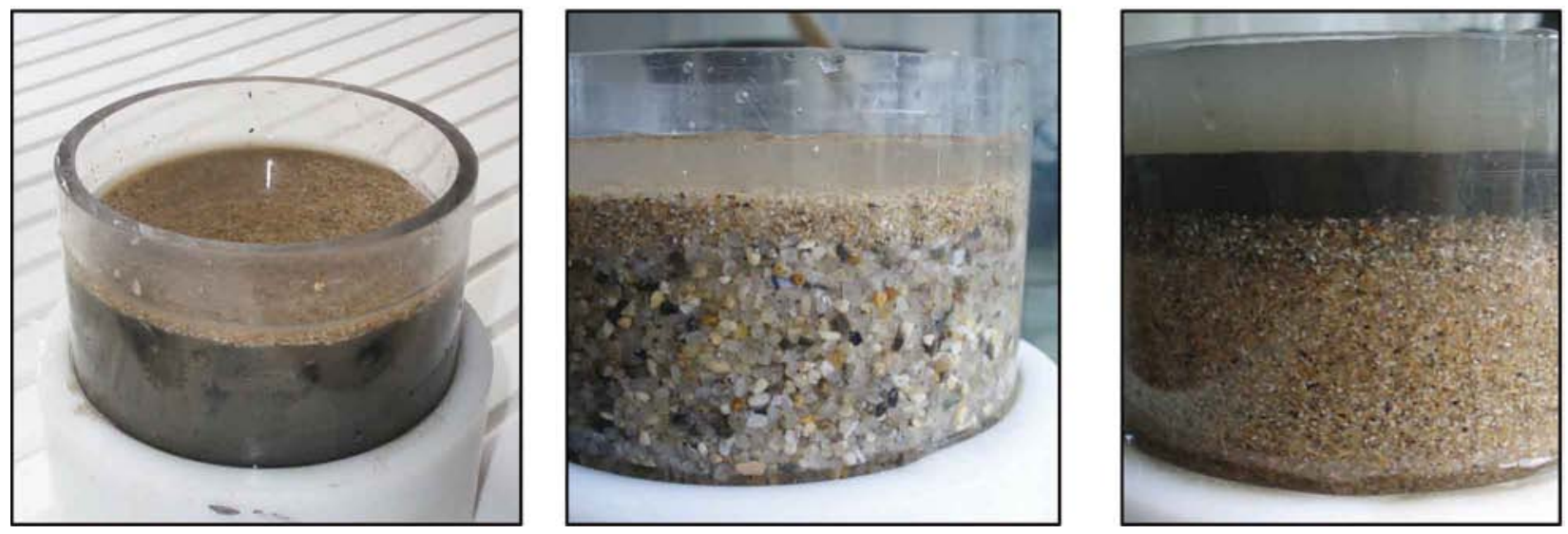

Figure 5 : Exemples d'interfaces reconstituées et testées en laboratoire avec ALTUS

signal rétrodiffusé, il était possible de mettre en évidence des caractéristiques physiques des divers faciès.

La description des tests et les résultats sont commentés dans [6]. Ils ont portés sur 5 faciès différenciés depuis les sables très grossiers jusqu'à la vase fine, en passant par des sables grossiers $\left(D_{50}: 497 \mu \mathrm{m}\right)$ et des sables fins $\left(D_{50}: 192\right.$ $\mu \mathrm{m})$. Pour la vase, deux teneurs en eau ont été testées afin d'obtenir une interface type " crème de vase » et une interface de vase consolidée.

La précision de la détection de l'interface eau-sédiment mise en évidence lors de ces tests en laboratoire est, en moyenne, de $0.8 \mathrm{~mm}$; elle est meilleure pour un sable fin et moins bonne $(1 \mathrm{~mm})$ pour une couche de type crème de vase. Cette précision peut être portée à $3 \mathrm{~mm}$ lorsqu'elle est affectée par le facteur température qui modifie la célérité de l'onde dans le milieu.

La connaissance du type de sédiment de surface est également importante vis-à-vis de la célérité de propagation. Les valeurs couramment utilisées sont : $1500 \mathrm{~m} / \mathrm{s}$ pour une vase fluide, $1640 \mathrm{~m} / \mathrm{s}$ pour un sable fin, $1780 \mathrm{~m} / \mathrm{s}$ pour un sable grossier et $1870 \mathrm{~m} / \mathrm{s}$ dans le cas de sables très grossiers.

Les principales constatations portant sur la détermination de l'épaisseur du dépôt supérieur sont :

dans le cas de faciès sableux : une très bonne détection de l'interface sable/sable pour des dépôts supérieurs de 5 à $20 \mathrm{~mm}$, à condition que les distributions granulométriques soient très tranchées : sable fin / sable grossier à très grossier. Une mauvaise pénétration du signal est relevée si ces distributions sont proches (de type sable fin / sable moyen ou sable grossier / sable très grossier)

dans le cas de faciès vaseux : une très bonne détection (erreur de 0.1 à $2 \mathrm{~mm}$ ) lorsqu'une couche type crème de vase (CV), d'épaisseur 5 à $20 \mathrm{~mm}$, surmonte une vase consolidée

dans le cas de sédiments mixtes : (i) mauvaise détection d'une CV d'épaisseur inférieure à $10 \mathrm{~mm}$ surmontant un sable, (ii) bonne détection d'une couche $(<10 \mathrm{~mm})$ de sable 
très grossier sur une vase consolidée ; détection défectueuse par contre si ce sable est fin à grossier.

\section{V — CONCLUSIONS ET PERSPECTIVES}

Le système ALTUS a été élaboré pour acquérir des données précises de variations de niveau du sédiment, que ce dernier soit vaseux, sableux ou un mélange de ces deux faciès. La possibilité d'acquérir avec ALTUS les données de caractéristiques de vagues (grâce à l'extension de la mémoire), en complément de ces données altimétriques, permet d'interpréter avec plus de précision la dynamique des sédiments (notamment quant aux forçages à l'origine de cette dynamique).

Par expérimentations en laboratoire, à partir de dépôts bien contrastés tels que : crème de vase sur vase consolidée, sables fins sur sables grossiers ou très grossiers de même que crème de vase sur sables, il est possible de quantifier avec une précision millimétrique, une épaisseur du dépôt supérieur de l'ordre de 5 à $20 \mathrm{~mm}$. Sur le terrain, cette précision de la mesure reste pour le moment tributaire de l'identification des faciès, la célérité du son étant pour partie, dépendante de ceux-ci.

Si des expérimentations complémentaires s'avèrent encore nécessaires pour constituer une " classification type " en fonction des signaux reçus par l'ALTUS, ces premiers résultats, concernant la détermination des types de faciès, sont néanmoins prometteurs.

Les développements futurs envisagés du système ALTUS portent (i) sur les possibilités d'enregistrer des signaux de meilleure qualité, (ii) sur l'utilisation d'un algorithme de classification qui soit validé sur un nombre important de faciès sédimentaires, apportant ainsi une contribution à la détermination de la nature des dépôts superficiels. Toutefois une validation par prélèvements in situ restera nécessaire.

\section{RÉFÉRENCES}

[1] Jestin H., Bassoullet P., Le Hir P., L'yavanc J., Degres Y. (1998) - Development of ALTUS, a high frequency acoustic submersible recording altimeter to accurately monitor bed elevation and quantify deposition or erosion of sediments. Oceans'98, Nice, France. Conference Proceedings. 1/3 189194

[2] Bassoullet P., Le Hir P., Gouleau D., Robert S. (2000) Sediment transport over an intertidal mudflat : field investigations and estimation of fluxes within the "Baie de MarennesOleron » (France). Continental Shelf Research. 20 (12/13) $1635-1653$

[3] Deloffre J., Verney R., Lafite R., Lesueur P., Lesourd S., CundY A. B. (2007) - Sedimentation on intertidal mudflats at the mouth of macrotidal estuaries : rhythms and preservation. Marine Geology. 241 19-32

[4] Verney R., Deloffre J., Brun Cottan J. C., Lafite R. (2007) - The effect of wave-induced turbulence on intertidal mudflats : Impact of boat traffic and wind. Application to the macrotidal Seine estuary (France). Continental Shelf Research. 27 594-612

[5] Soulsby R. L. (1997) - Dynamics of marine sands. A manual for practical applications. London, Thomas Telford. $249 \mathrm{p}$

[6] Verney R., Voineson G., Jestin H., Bassoullet P., Le Hir P. (2009) - Laboratory investigation of sediment bed features with a submersible acoustic echosounder : the ALTUS system. INTERCOH'09, Rio de Janeiro, Brésil, 3-8 May, abstracts book. 185-186 